\title{
Circulating tumor cell clusters: What we know and what we expect (Review)
}

\author{
YUPENG HONG $^{1}$, FRANCIA FANG ${ }^{2}$ and QI ZHANG $^{3}$ \\ ${ }^{1}$ Department of Oncology, Zhejiang Provincial People's Hospital, Hangzhou, Zhejiang 310014, \\ P.R. China; ${ }^{2}$ Trinity College of Arts and Sciences, Duke University, Durham, NC 27710, USA; \\ ${ }^{3}$ Department of Hepatobiliary and Pancreatic Surgery, The Second Affiliated Hospital, \\ Zhejiang University School of Medicine, Hangzhou, Zhejiang 310009, P.R. China
}

Received August 16, 2016; Accepted October 14, 2016

DOI: $10.3892 /$ ijo.2016.3747

\begin{abstract}
The major cause of cancer-associated mortality is tumor metastasis, a disease that is far from understood. Many studies have observed circulating tumor cells (CTCs) in patients' circulation systems, and a few latest investigations showed that CTC clusters have a potentially high capacity of metastasis. The capture and analysis of CTC clusters offer new insights into tumor metastasis and can facilitate the development of cancer treatments. We reviewed the research history of the CTC clusters, as well as the technologies used for detecting and isolating CTC clusters. In addition, we discuss the characteristics of CTC clusters and their roles in tumor dissemination. Clinical relevance of CTC clusters was also implicated in currently limited data. Moving forward, the next frontier in this field is to develop more efficient capture methods and decipher conundrums of characterization of CTC clusters. This will ultimately identify the clinical value of CTC clusters as a biomarker and therapeutic target.
\end{abstract}

\section{Contents}

1. Introduction

2. The discovery history of CTC clusters

3. Methods for CTC cluster isolation, capture and identification

4. Biological significance of CTC cluster and its role in tumor metastasis

5. Clinical application of CTC clusters

6. Perspectives

Correspondence to: Dr Qi Zhang, Department of Hepatobiliary and Pancreatic Surgery, The Second Affiliated Hospital, Zhejiang University School of Medicine, 88 Jiefang Road, Hangzhou, Zhejiang 310009, P.R. China

E-mail: zhangqi86@zju.edu.cn

Key words: CTC cluster, CTC microemboli, CTC capture, metastasis, clinical application, translational medicine, phenotype

\section{Introduction}

The vast majority of cancer-related deaths are caused by metastasis, the dissemination of tumor cells from their original sites to distant organs mainly through the blood circulation system $(1,2)$. Unfortunately, after decades of exploration, our understanding of tumor metastasis is still far from complete, let alone enough for prevention and cure. Circulating tumor cells (CTCs) have recently been attracting great attention due to their key role in tumor metastasis, even though they were first discovered almost 150 years ago $(3,4)$. Given the value of prognosis prediction of CTCs, the US Food and Drug Administration (FDA) has approved their clinical use in metastatic breast, colorectal and prostate cancers (5). CTCs can be frequently and conveniently detected and are valuable for personalized treatment. Increasing studies have proved the value of CTC detection in a number of different types of cancer (5-9); however, great efforts are needed to decipher the clinical implication underlying CTCs.

Additionally, referred to as circulating tumor microemboli, circulating micrometastases or circulating tumor aggregates, CTC clusters are defined as groups of tumor cells (more than two or three cells, varied among studies) that travel together in the bloodstream (10). Early in the 1970s, a series of preclinical studies demonstrated that CTC clusters had a greater predisposition of forming distal metastasis than single CTCs (11-15). However, further studies were stagnant for decades due to limitations of CTC cluster isolation in human. In spite of great advances on CTCs recently, little progress has been achieved. For instance, investigators have already recognized that CTCs are heterogeneous, with certain subgroups of CTCs harboring higher metastatic potential (16-18).

The prevalence and amount of CTC clusters can be underestimated due to their short detection window and lack of appropriate detection methods. On one hand, the life span of CTC clusters in circulation is extremely short [much shorter than individual CTCs, which can also exist in the circulation for only several hours (19)] due to their interception by small vessels. On the other hand, current technologies are responsible for the underrating of CTC clusters because few specialized devices exist for the detection of CTC clusters. 
The relative rarity and incompetence of existing capture methods limit our knowledge of CTC clusters, thus many questions are awaiting to be answered, including where CTC clusters come from, what they consist of, and how they take advantage to form metastases. To resolve these problems, acquirement of enough viable CTC clusters is the key, which makes subsequent molecular, genetic and functional experiments possible. In this review, we discuss the valuable knowledge of CTC clusters from all relevant aspects including discovery history, detection and isolation methods, pathophysiological characteristics as well as their clinical implications.

\section{The discovery history of CTC clusters}

Similar to the long history of CTCs, the first recognition of CTC clusters can be dated back to 1950s (Fig. 1). In 1954, Watanabe highlighted the role of CTC clusters in tumor metastases formation by injecting bronchogenic carcinoma cells from jugular veins of mice, showing that viable tumor cells in clusters formed metastases, unlike individual cells (20). Studies in the following two decades confirmed this result on both melanoma-derived lung metastases and colon cancer-derived liver metastases models $(11,15,21,22)$ and also illustrated that the metastases formation partially depended on the size and concentration of CTC clusters $(13,23)$. Although cancer cells intercepted by vessels were seen in animal models decades ago, the tumor cell emboli entrapped in vessels in human were proved most recently in the microvasculature of the lungs in the 3 out of 8 patients with metastatic breast and cervical carcinomas (24).

Pioneers in the field also studied the relationship between CTC clusters and the sites of metastases formation. Liotta et al revealed that the size distribution of vessels was an important determinant of the distribution and survival of CTC clusters in the circulation system (14). In addition, some investigators found that CTC clusters of different tumor cells harbored different metastasis proclivity (25).

An attempt was also made to explore the mechanisms of how CTC clusters possess survival and metastasis advantages. Recent studies imply that CTC clusters have their specialized microenvironments and are not simply an aggregation of tumor cells (26). Interaction between tumor cells and accessory cells was found to provide tumor cells with survival advantages via different ways, although the detailed mechanisms required in-depth investigation $(16,27,28)$. Nowadays, with the improvement of CTC cluster isolation technology, other physical properties of CTC clusters such as density and electromechanical characteristics have been under assessment and we can soon expect deeper understanding of these aspects.

Despite the long history in this field, information surrounding CTC clusters remain largely unknown. Increased efforts are urgently required to characterize CTC clusters and fully understand their roles in tumor metastasis, both clinically and mechanically.

\section{Methods for CTC cluster isolation, capture and identification}

Currently, very few methods have been developed for specialized detection of CTC clusters. In most cases, CTC clusters were incidentally observed when detecting individual CTCs. The devices used for CTC isolation and capture are based on the differences in physical properties (e.g., density, size, deformability, electric charges), and biological properties (e.g., antigen expression) between CTCs and non-tumor cells. Currently, limited data of CTC clusters in patients vary greatly according to tumor type, disease stage, detection platform, and other factors (Table I). However, these existing platforms are not ideal for CTC cluster isolation since they usually underestimate the amount of CTC clusters. Thus, it is important to approach with caution when interpreting the results of CTC clusters derived from single CTC specialized isolation platforms.

Antibody-based methods. Antibody-based methods are the most widely used capture techniques for CTC clusters. The antibodies are mainly pertained to epithelial cell surface markers that are absent from blood or stroma cells. Among these antibodies, the epithelial cell adhesion molecule (EpCAM) is the most commonly used. In CellSearch ${ }^{\circledR}$, the first and only commercial CTC isolation platform approved by the US FDA, a CTC cluster is defined as a group of CTCs containing three or more cells expressing EpCAM and cytokeratins (CKs) without expression of CD45 and has (4',6-diamidino-2-phenylindole)stained nuclei (29). Blood-spiking experiments with $\mathrm{HeLa}$ cells expressing histone H2B-GFP confirmed that CTC clusters were not artifacts caused by sample manipulation (10). Although CTC clusters were rarely captured by CellSearch in a majority of solid tumors, they were frequently detected (25/97) in small cell lung cancer (SCLC) and indicated poor prognosis (10). As a representative of high-throughput microfluidic devices, herringbone (HB)-chip was developed to improve capture efficiency by using microvortices to increase the interaction between tumor cells and antibodies (26). The number of tumor cells in a CTC cluster captured by HB-chip ranged from 4 to 12 ; however, CTC clusters were only found in 3 out of 19 patients with metastatic prostate or lung cancer (26). In order to further identify invasive CTC clusters, a platform was developed to detect CTC clusters that uptake cell-adhesion matrix, and $17 \%$ of samples were found positive in patients with metastatic castration-resistant prostate cancer (30).

Antibody-conjugated magnetic microbeads or nanoparticles binding to a specific surface antigen such as CD45 were also used for isolating CTCs and CTC clusters (31-34). After incubation, the blood sample was exposed to a non-uniform magnetic field to isolate the labeled cells. In a study using $\mathrm{CK}$ and prostate specific antigen as conjugated biomarkers in prostate cancer, the prevalence of CTC clusters was as high as $80 \%$ (32). In another study for colorectal cancer, the immunomagnetic labeled CK antibodies could separate CTC clusters from the peripheral blood in $68.8 \%$ of patients (33). However, certain investigators used a negative selection strategy to remove non-tumor cells from CTCs and CTC clusters, and found that the prevalence of CTC clusters was rare (31). In general, the efficiency of immunomagnetic methods mainly depends on two factors: i) the expression and specificity of the target antigen and the affinity between antigens and antibodies; and ii) the efficiency of immunomagnetic labeling process and magnetic particles. 

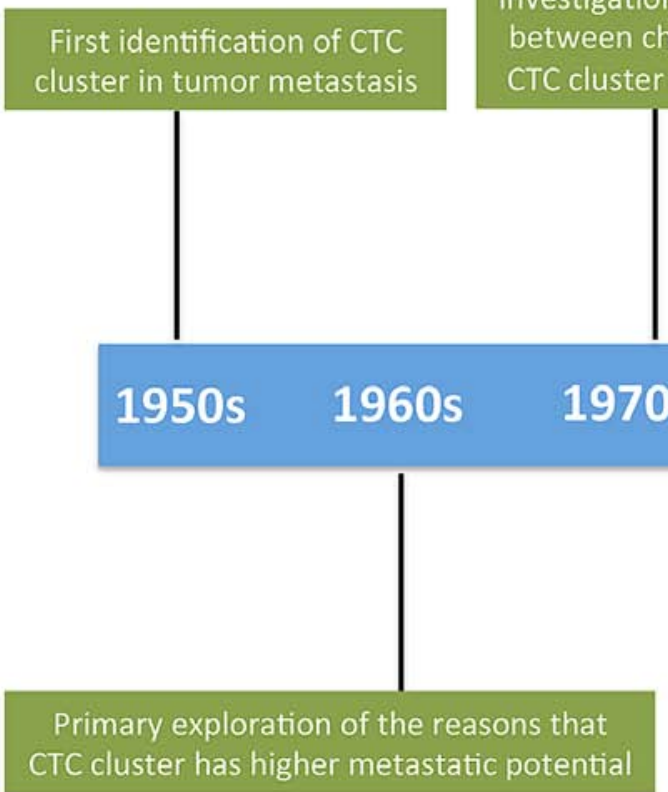

Investigation of relationship

between characteristics of

CTC cluster and metastasis
Detection of CTC

cluster using isolation

methods for CTC
First device for specialized CTC cluster isolation

Figure 1. The milestones of CTC cluster discovery and isolation.

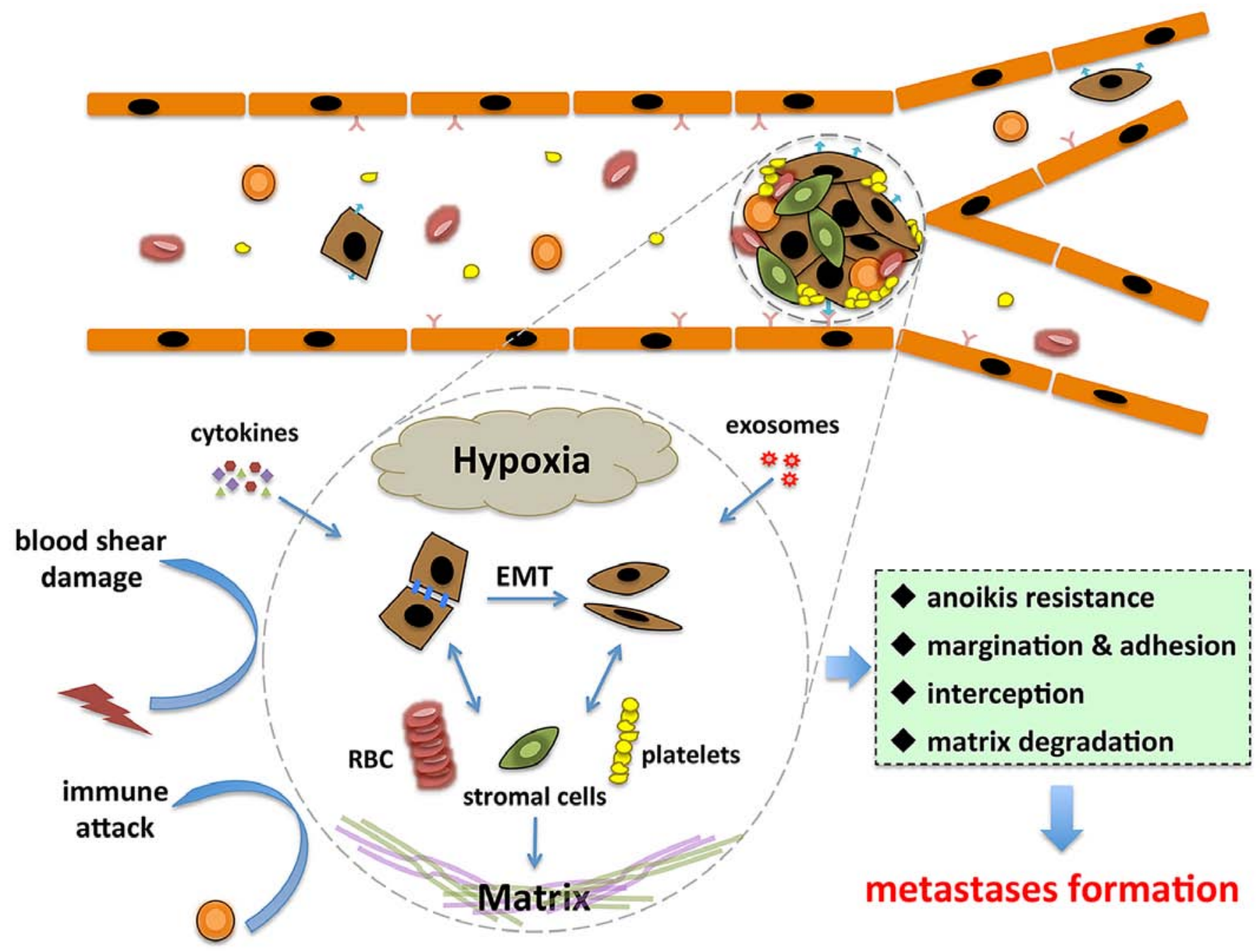

Figure 2. The CTC clusters merit higher potential of metastasis. In the circulation system, the hypoxic microenvironment of CTC cluster comprises of CTC cells, mesenchymal cells, epithelial cells, pericytes, immune cells, platelets, and cancer-associated fibroblasts would have some interactions with cytokines and exosomes. Such microenvironment protects CTC clusters from blood shear force damage and immune attack. 


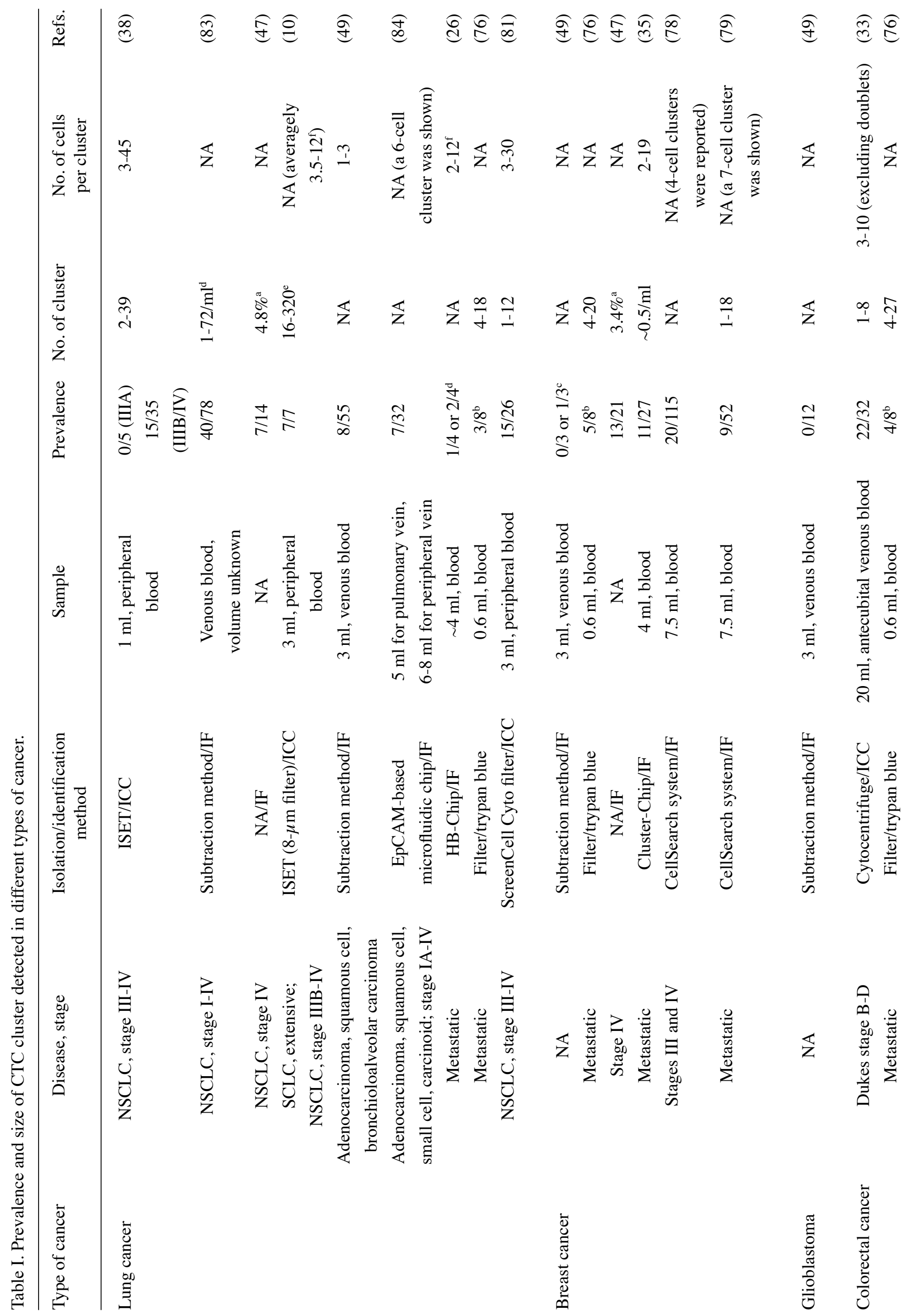




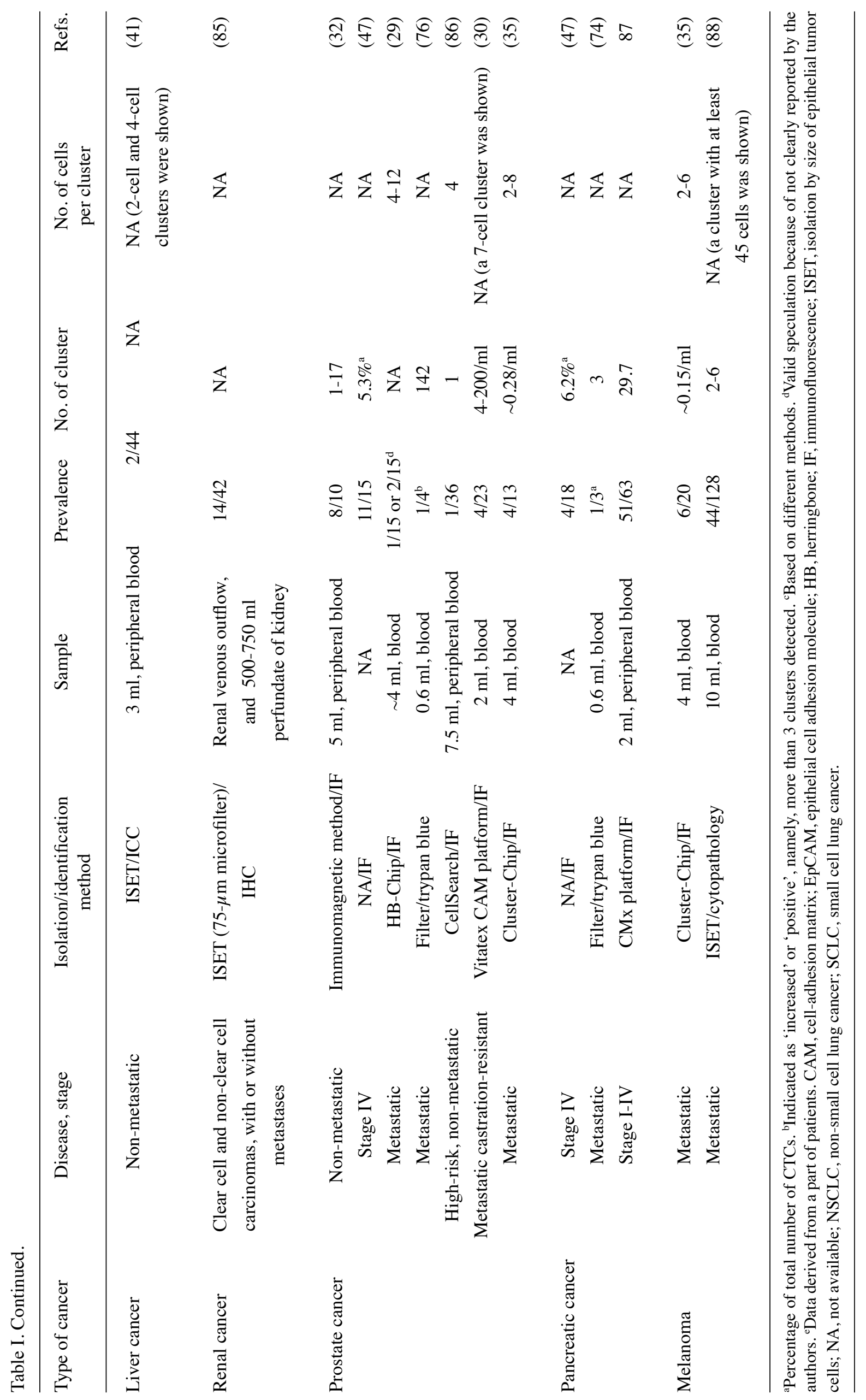


As a by-product of CTC detection, CTC clusters are insufficiently detected by antibody-based methods for several reasons. Compared to single CTCs, CTC clusters have smaller surface-area-to-volume ratios, which reduce the efficiency of antibody capture. This situation can be more obvious in larger CTC clusters. In addition, the integrity of CTC clusters is prone to be impaired in devices with turbulent flow, which can result in an anamorphic number and size of CTC clusters (35). Other limitations of antibody-based methods for single CTC detection are also applicable to CTC clusters, such as the limited expression of target antigens (35).

Physical property-based methods. Physical property differences in cell density, size, dielectric properties and mechanical plasticity, can all be utilized to isolate CTCs and CTC clusters. For instance, size-based isolation relies on the larger size of CTCs compared to that of blood cells. This has been achieved by using track-etched polycarbonate filters, which are porous membranes containing numerous randomly distributed $8-\mu \mathrm{m}$-diameter, cylindrical pores (36). Isolation by size of epithelial tumor cells (ISET) platform seems more reasonable for CTC cluster isolation since the difference between CTC clusters and non-tumor cells is more significant. One study reported that 2 out of 23 patients with primary liver cancer were positive for CTC clusters using an ISET platform (37). Another study using a similar ISET platform isolated CTC clusters from all patients with lung cancer (29). As an unbiased method, ISET was believed to be more sensitive than antibodybased methods. For example, CTC clusters were observed in $43 \%$ of patients with non-small cell lung cancer (NSCLC) using ISET but were completely undetectable by CellSearch (38). Generally, the ISET platform holds a few advantages: i) it retains the natural status of CTC clusters without the binding of antibodies or nanoparticles; ii) it allows direct filtration of peripheral blood without preprocessing; iii) it can maintain the integrity of CTC clusters; iv) it is cost-friendly compared to antibody-based methods.

Physical property-based methods of CTC cluster isolation can be also combined with and strengthened by microfluidic technology (39-44). By taking advantage of centrifugal forces which can facilitate CTC and CTC cluster separation, a two-inlet, two-outlet spiral microchannel with a total length of $10 \mathrm{~cm}$ was designed (45). This device is able to continuously collect viable CTCs and CTC clusters allowing simple coupling with a convectional 96-well plate for subsequent biological assays, and CTC clusters with size of 50-100 $\mu \mathrm{m}$ in patients were successfully detected (45). Harouaka et al developed a new flexible micro spring array (FMSA) device for enrichment of viable CTC clusters according to their sizes. The FMSA device was based on flexible structures at micro scale that minimized cell damage and could preserve cell viability while maximizing throughput to allow for rapid enrichment directly from blood samples without sample preprocessing. CTC clusters with 2-20 tumor cells were detected in patients with breast, lung, and colorectal cancer using the FMSA device (46). Extraordinarily, the first attempt for specific isolation of CTC clusters was achieved in 2015. The Cluster-Chip, based on microfluidic and antigen-independent technologies, is able to isolate CTC clusters through specialized bifurcating traps under low-shear stress conditions that preserve their integrity.
Even two-cell clusters can be efficiently captured using this technique (35). The chip comprises of a set of triangular pillars and captures CTC clusters by relying on the strength of cell-to-cell junctions as clusters flow through the pillars at physiological speed. This model is designed to exclude two-cell clumps with a loosened combination, which may occur in incidentally attached cells. Cluster-Chip was able to find CTC clusters in 30-40\% of patients with metastatic breast, prostate cancer or melanoma (35).

Additional innovative detection strategies for CTC clusters. Some additional approaches have been developed to detect CTC clusters by taking advantage of the physical and biological properties of epithelial cells. High-resolution imaging combined with enrichment methods was used to isolate CTC clusters. A group of investigators separated CK-positive, CD45-negative CTC clusters, which were then analyzed by a hematopathologist. In their report, CTC clusters were detected in $93,54,50$ and $22 \%$ of patients with prostate cancer, breast cancer, NSCLC, and pancreatic cancer, respectively (47). Another study reported a novel integrated cellular and molecular approach of subtraction enrichment and immunostaining-fluorescencen in situ hybridization (48). The integrated platform depleted white blood cells and red blood cells and established an expeditious detection of non-hypotonic damaged and non-hematopoietic CTC clusters, regardless of CKs or EpCAM expression or size variation. This platform was able to efficiently detect, isolate, and characterize CTC clusters from various types of cancer including lung cancer, glioma and melanoma (48).

Special methods for CTC cluster identification. Theoretically, it is difficult to judge whether an individual cell is a tumor cell or not. This dilemma also exists in the identification of CTC clusters. Most researchers prefer to use CKs as tumor markers. Some investigators adapted fluorescence in situ hybridization with the centromere of chromosome 8 (CEP8), since more than 2 hybridization signals of CEP8 indicates chromosomal variation and the cell is expected to be malignant (49). Aptamers specifically selected from postoperative tumor tissue were also used for tumor cell identification, and were able to detect more CTC clusters than CKs (50).

\section{Biological significance of CTC cluster and its role in tumor metastasis}

Beyond the enumeration of CTC clusters, their molecular characterization offers insights into their origins and metastatic potential. It may also provide clues to their evolution during the course of cancer treatment and the mechanisms of treatment resistance relevant to CTC clusters. However, CTC clusters with different sizes or components have distinct biological and physical characteristics. Specifically, King et al systematically studied the physical features of CTC clusters that consisted of 2-5 tumor cells, which provided a straightforward view of the enhanced metastatic potential of CTC clusters (51).

Origins of CTC clusters. The origins of CTC clusters and the way their integrity is preserved are both interesting to note. CTC clusters can directly be derived from primary tumors or 
from the aggregation/proliferation of single CTCs. Current evidence acquired from breast cancer remains limited, and excludes intravascular aggregation of CTCs as a main cause of CTC clusters (16). In parallel with this, we designed an in vitro platform to mimic blood stream, and the results showed that the aggregation/proliferation of single CTCs was impossible because of the sheer force of blood (unpublished data). However, the aggregation and proliferation were still possible when CTCs were located in inflammatory sites or when small vessels intercepted CTCs.

Differently originated CTC clusters may have different formation mechanisms and possess different biological characteristics. The molecules responsible for tumor cell aggregation within a CTC cluster are currently under investigation and could be good targets for treatment. At least in breast cancer, plakoglobin and keratin 14 that are both associated with desmosomes and hemidesmosomes have been found to be critical for CTC cluster formation. Inhibition of these proteins reduced CTC cluster formation and distal metastases $(16,52)$. Interactions between Thomsen-Friedenreich glycoantigen and galectin-3 also take part in breast cancer cell aggregation (53). In addition, some pro-inflammatory cytokines such as interleukin- 6 and tumor necrosis factor- $\alpha$ could also promote tumor cell growth as clusters and induce adhesive recruitment in the circulation system (54). For patients of metastatic stage, the existence of CTC clusters from metastases is possible and makes things more complicated. The dynamic change of CTC clusters in the circulation system is also mysterious because it is difficult to monitor CTC clusters in vivo. In the future, more investigations are warranted to solve these specific problems, and should then be directed towards finding targeted therapeutic approaches that can be performed to lower the risk of CTC clusters and improve cancer treatments.

Internal characteristics of CTC clusters. Some studies have demonstrated that CTC clusters are comprised of a number of tumor cells with or without non-tumor cells such as mesenchymal cells, epithelial cells, pericytes, immune cells, platelets, and cancer-associated fibroblasts (16,27,55-62). These non-malignant components contribute to the survival and metastatic advantages of CTC clusters in various ways. For instance, the presence of heterotypic tumor-derived stromal cells (e.g., fibroblasts, endothelial or tumor-infiltrated myeloid cells) increased the viability of tumor cells within CTC clusters, and facilitated metastases formation (27). However, when the cancer-associated fibroblasts were partially depleted, a significantly decreased number of metastases was observed (27). Another study using tumor cell and endothelial cell co-culture in a mouse model found that tumor cells were more potent in promoting angiogenesis in the presence of endothelial cells and resulted in increased numbers and larger size of metastases (28). Promotion of metastasis was also associated with the interplay between tumor cells and non-tumor cells such as platelets and leukocytes within CTC clusters $(56,57,59,63)$. Platelets in the CTC clusters are believed to protect tumor cells from blood shear damage and immune attacks by physically shielding other complex influences via paracrine signaling and direct contact (64). Furthermore, some undefined cells such as CK-positive dendritic-like cells were also found in CTC clusters, but the nature and significance were unknown (33).
CTC clusters also show mesenchymal traits (63). The epithelial-mesenchymal transition (EMT) status of tumor cells within a CTC cluster is convincing, and is more obvious than that of single CTC (29). For example, at least one third of tumor cells in the clusters were $\mathrm{CK}$ negative in colorectal cancer (33). EpCAM and CK were both heterogeneously expressed in CTC clusters derived from NSCLC patients (29). Specifically, a majority of isolated CTC clusters derived from metastatic NSCLC patients harbored dual epithelial-mesenchymal phenotype, suggesting that EMT is a relevant process for metastasis caused by CTC clusters (65). Several hypotheses have been proposed to explain the EMT status in CTC clusters. For instance, the expression of mesenchymal markers in a CTC cluster could result from proliferation of a single cell that had undergone EMT, or from the mesenchymal transformation of a pre-existing CTC cluster in the circulation system (63).

Whether CTC clusters are of oligoclonal/polyclonal or monoclonal origin is also under investigation. In breast and pancreatic cancers, oligoclonal/polyclonal rather than monoclonal CTC clusters were observed by assessing lung metastases in a mouse model $(16,66)$. Although CTC clusters have up to 100-fold increased metastatic potential compared to individual CTCs (67), it remains uncertain whether the tumor cells within a CTC cluster have identical metastatic potentials. Intravenous injections of CTC clusters comprised of two cell lines with distinct metastatic potentials resulted in lung metastases with the metastatic unique cell line karyotype (68). This finding suggested that the presence of metastatic cells did not change the inability of non-metastatic cells to form a distant organ, and implied that different tumor cells within the same cluster maintained their own metastatic potential (68). Accordingly, the metastatic potential of a CTC cluster may be dependent on the most malignant tumor cells. However, contradictory results were provided by another study, in which two cell lines that harbored different metastatic potential were mixed and injected into the flank of nude mice (58). Intriguingly, in addition to the polyclonal primary tumor at the inoculation site, the CTC clusters and almost $90 \%$ of the lung metastases were also found to be polyclonal (58). When the cell lines were injected respectively, merely $10 \%$ of the metastases arose from the less metastatic cell line, suggesting that tumor cells with lower metastatic potential acquired higher metastatic capability when cooperating with other cell lines in the CTC clusters (58).

In summary, clear evidence has shown eminent heterogeneity in CTC clusters and their marked complex compositions. Future studies should pay close attention to the dynamic change of the composition of CTC clusters, crosstalk between different compositions, and their roles in metastasis.

Metastasis-associated features of CTC clusters. Current studies have partially elucidated the reasons for CTC clusters to have higher potential of metastasis (Fig. 2). First, tumor cells within CTC clusters showed prolonged survival and decreased apoptosis (29). Mechanically, CTC clusters prevent tumor cells from anoikis through interaction between circulating galectin-3 and cancer-associated mucin1 (MUC1), also promoting the formation and survival of CTC clusters in the circulation system (69). The elevated galectin-3 in the circulation also facilitate tumor cancer adhesion to endothelial 
Table II. Association between CTC cluster and clinical characteristics.

Type of cancer $\quad$ Clinical relevance Refs.

Prostate cancer Antioxidant gene expression level in CTC clusters could be
used for tumor diagnosis and recurrence prediction

Breast cancer The presence of CTC cluster was associated with worse PFS

The presence of CTC cluster impaired prognosis

Renal cancer The presence of CTC cluster was correlated with larger primary tumor, with higher frequency of lung metastasis, and with micronodular phenotype in the primary tumor

SCLC The presence of CTC cluster was associated with shorter PFS and OS

NSCLC

The presence of CTC cluster was not associated with histological subtype

The presence of CTC cluster was not associated with tumor type or stage

No significant difference between numbers of CTC cluster and disease stage

Liver cancer

The presence of CTC cluster was association with tumor diffusion, portal tumor thrombosis, and shorter survival

Colorectal cancer Elevation of CTC cluster number was correlated with macroscopic progression, and the numbers of cancer cells in the CTC clusters were significantly higher in non-responder after chemotherapy

Pancreatic cancer PFS and OS were significantly shorter in unfavorable group ( $>30 / 2 \mathrm{ml})$ compared to those of favorable group (<30/2 ml). CTC cluster was an independent prognostic factor for PFS and OS

Melanoma

OS was significantly decreased in patients with CTC clusters, independent of treatment

CTC, circulating tumor cell; NSCLC, non-small cell lung cancer; OS, overall survival; PFS, progression-free survival; SCLC, small cell lung cancer.

cells by interacting with MUC1 on tumor cell surface $(70,71)$. These findings deepen our understanding of the molecular mechanisms of CTC cluster dissemination and suggests that interference of this interaction may provide a novel therapeutic approach for preventing metastasis. Moreover, accessory cells such as fibroblasts, endothelial cells, platelets, and immune cells can form a niche $(27,72,73)$, which is beneficial for tumor cells in CTC clusters when facilitating metastasis $(27,73,74)$. For instance, platelets induce tumor cells to undergo EMT via TGF- $\beta /$ Smad and NF- $\kappa$ B pathways by secretion of TGF- $\beta$ and direct interaction with tumor cells (72).

Second, the physical specialty of CTC clusters allows for a greater likelihood of it residing in distant organs. Microvasculature of viscera can retain large CTCs, thus it can retain CTC clusters more easily (24). Compared to single CTCs, CTC clusters have larger sizes and a slower travelling velocity, making them easier to be intercepted by small vessels (75). In traditional concepts, interaction of tumor cells with vascular endothelial cells makes up the premise of the extravasation of tumor cells. From a physical biology standpoint, it has been reported that CTC clusters tended to undergo margination, rotation, and adherence to endothelium via interaction with E-selectin (51). CTC clusters have a much lower rolling velocity than individual CTCs, and are susceptible for margination and attachment to vascular wall even in vessels with diameters not small enough to intercept clusters
(51). In addition to sphere-like clusters, various appearances of CTC clusters associated with the collective migration of tumor cells were observed (29). Most noticeably, CTC clusters with linear or triangular geometry showed no difference in velocity along the vessel (51). These clusters also provide a special microenvironment for the tumor cells within them, and this microenvironment varies according to different sizes and constitutions of the clusters. CTC clusters with bigger sizes have significant hypoxic microenvironments compared to those with smaller sizes and single CTCs (76).

In addition, cytokines such as interleukin- 6 and tumor necrosis factor- $\alpha$ can induce a positive feedback loop, leading to the aggregation of subsequent tumor cells and adhesion of tumor cells to endothelium (54). Alternatively, intravascular metastatic formation has also been proposed in certain cases in order to better understand tumor metastasis $(49,53,77)$. CTC clusters seem more likely to take this strategy due to their large size and survival advantage in vessels. However, this assumption is challenged by a recent study. By using microfluidic devices and zebrafish models, Au et al discovered that CTC clusters containing $\leq 20$ cells were able to pass through capillary-sized vessels by reorganizing into single-file chain-like geometries (67) Astonishingly, the shape of these CTC clusters is highly plastic, and the cells are able to easily reorganize again into a sphere-like cluster after having traversed the capillaries (67). From another view, 
these findings provide us with a better understanding of CTC cluster-based metastasis.

\section{Clinical application of CTC clusters}

As a minimally invasive technique with potential roles in diagnosis, decision-making, treatment assessment, and relapse monitoring in patients, CTCs have been involved in many preclinical studies and clinical trials. Particularly, investigators have explored the clinical implications of CTC clusters (Table II). The presence of CTC clusters in patients is a harbinger of bad prognosis, and certain studies have even identified an association between CTC clusters and survival. One such study enrolled 97 patients with SCLC and found that $32 \%$ of patients were positive for CTC clusters, and the presence of CTC clusters at baseline indicated shorter progression-free survival and overall survival (11). An additional prognostic value of CTC clusters in patients with elevated CTCs were reported in two prospective cohorts of advanced stage or metastatic breast cancer $(78,79)$. Furthermore, information regarding CTC clusters were indicative of the failure of chemotherapy treatments in colorectal cancer (60). Similarly, CTC clusters also had predictive values in NSCLC patients (38). It was demonstrated that the prevalence of CTC clusters increased in NSCLC of higher stages (10/23 in stage IV, 5/12 in stage IIIB, and 0/5 in stage IIIA) (38). Unfortunately, there was no association found between histological subtypes and the presence of CTC clusters (38). Likewise, CTC clusters can be detected in more than half of 28 consecutive patients with stage III-IV lung cancer, but no correlation between the number of CTC clusters and tumor type or stage was observed (80). Moreover, the association between abundance of CTC clusters and disease stage also failed to be detected in colorectal cancer, though a trend of CTC cluster elevation was noted in patients with higher stage (33).

Gene expression and changes of CTC clusters during treatment were also studied. In patients with prostate cancer, the antioxidant gene expression level had excellent prognostic and predictive value, and could be used for evaluation of therapy and monitoring of tumor recurrence (81). Although treatments may influence the stability of CTC clusters, chemotherapy was incapable of destroying all CTC clusters. A higher reproducible rate of CTC clusters than that of single CTCs was found during the follow-up of patients after adjuvant chemotherapy (33). Similarly, the reduction of the proportion of CTC clusters was not significant even though the total number of CTCs was dramatically reduced (75), suggesting that CTC clusters may have different clinical relevance with single CTCs.

Although the importance of CTC clusters is gradually accepted, few effects have been made in cancer treatment by targeting CTC clusters. Recently, a Korean group succeeded in prolonging survival in a mouse model of breast cancer by dissociating CTC clusters using urokinase (82), making a first step in CTC cluster-relevant treatment. However, because the dissociated CTCs can still form metastases, the effects of such strategy may be mild and needs further evaluation.

In conclusion, the clinical value of CTC clusters currently remains elusive. Further diligent study is necessary in order to exploit the full potential of CTC clusters in clinical applications.

\section{Perspectives}

The molecular characterization of CTC clusters may revolutionize our interpretation of cancer metastasis. However, many questions need to be answered before CTC clusters can begin making sense for biological understanding and clinical use. First, methods optimized for specific isolation of CTC clusters preserving their original status are required due to the lack of an efficient or widely approved detection platform that does not hinder in-depth study of CTC clusters. Second, further identification is needed for the adhesion molecules responsible for CTC cluster formation, as well as the genetic and biological specialty of the tumor cells harboring these molecules. Third, the biological details of creation, travelling in the circulation, and metastases formation in distant organs of CTC clusters also demand further investigation. Finally, the clinical significance of CTC clusters is awaiting confirmation, and treatments based on CTC clusters may be promising to reduce metastasis events. Finding viable solutions to these problems will open up further fields of study for CTC clusters, and affirm its value in clinical practice spanning from early diagnosis of cancer to relapse monitoring.

\section{Acknowledgements}

This study was supported by the National Natural Science Foundation of China (81401954) and the Medical Science and Technology Program of Zhejiang Province, China (2015KYA114).

\section{References}

1. Krebs MG, Metcalf RL, Carter L, Brady G, Blackhall FH and Dive C: Molecular analysis of circulating tumour cells - biology and biomarkers. Nat Rev Clin Oncol 11: 129-144, 2014.

2. Siegel RL, Miller KD and Jemal A: Cancer statistics, 2015. CA Cancer J Clin 65: 5-29, 2015.

3. Lianidou ES: Circulating tumor cell isolation: A marathon race worth running. Clin Chem 60: 287-289, 2014.

4. Plaks V, Koopman CD and Werb Z: Cancer. Circulating tumor cells. Science 341: 1186-1188, 2013.

5. Cristofanilli M, Budd GT, Ellis MJ, Stopeck A, Matera J, Miller MC, Reuben JM, Doyle GV, Allard WJ, Terstappen LW, et al: Circulating tumor cells, disease progression, and survival in metastatic breast cancer. N Engl J Med 351: 781-791, 2004

6. de Bono JS, Scher HI, Montgomery RB, Parker C, Miller MC, Tissing H, Doyle GV, Terstappen LW, Pienta KJ and Raghavan D: Circulating tumor cells predict survival benefit from treatment in metastatic castration-resistant prostate cancer. Clin Cancer Res 14: 6302-6309, 2008.

7. Cohen SJ, Punt CJ, Iannotti N, Saidman BH, Sabbath KD, Gabrail NY, Picus J, Morse M, Mitchell E, Miller MC, et al: Relationship of circulating tumor cells to tumor response, progression-free survival, and overall survival in patients with metastatic colorectal cancer. J Clin Oncol 26: 3213-3221, 2008.

8. Cristofanilli M: Circulating tumor cells, disease progression, and survival in metastatic breast cancer. Semin Oncol 33 (Suppl 9): S9-S14, 2006.

9. Rhim AD, MirekET,Aiello NM,Maitra A,Bailey JM,McAllisterF, Reichert M, Beatty GL, Rustgi AK, Vonderheide RH, et al: EMT and dissemination precede pancreatic tumor formation. Cell 148: 349-361, 2012.

10. Hou JM, Krebs MG, Lancashire L, Sloane R, Backen A, Swain RK, Priest LJ, Greystoke A, Zhou C, Morris K, et al: Clinical significance and molecular characteristics of circulating tumor cells and circulating tumor microemboli in patients with small-cell lung cancer. J Clin Oncol 30: 525-532, 2012.

11. Fidler IJ: The relationship of embolic homogeneity, number, size and viability to the incidence of experimental metastasis. Eur J Cancer 9: 223-227, 1973. 
12. Lione A and Bosmann HB: Quantitative relationship between volume of tumour cell units and their intravascular survival. Br J Cancer 37: 248-253, 1978.

13. Liotta LA, Kleinerman J and Saidel GM: Quantitative relationships of intravascular tumor cells, tumor vessels, and pulmonary metastases following tumor implantation. Cancer Res 34: 997-1004, 1974.

14. Liotta LA, Saidel MG and Kleinerman J: The significance of hematogenous tumor cell clumps in the metastatic process Cancer Res 36: 889-894, 1976.

15. Thompson SC: The colony forming efficiency of single cells and cell aggregates from a spontaneous mouse mammary tumour using the lung colony assay. Br J Cancer 30: 332-336, 1974.

16. Aceto N, Bardia A, Miyamoto DT, Donaldson MC, Wittner BS, Spencer JA, Yu M, Pely A, Engstrom A, Zhu H, et al: Circulating tumor cell clusters are oligoclonal precursors of breast cancer metastasis. Cell 158: 1110-1122, 2014.

17. Chen JF, Ho H, Lichterman J, Lu YT, Zhang Y, Garcia MA, Chen SF, Liang AJ, Hodara E, Zhau HE, et al: Subclassification of prostate cancer circulating tumor cells by nuclear size reveals very small nuclear circulating tumor cells in patients with visceral metastases. Cancer 121: 3240-3251, 2015.

18. de Wit S, van Dalum G, Lenferink AT, Tibbe AG, Hiltermann TJ, Groen HJ, van Rijn CJ and Terstappen LW: The detection of $\operatorname{EpCAM}(+)$ and $\operatorname{EpCAM}(-)$ circulating tumor cells. Sci Rep 5: 12270,2015

19. Meng S, Tripathy D, Frenkel EP, Shete S, Naftalis EZ, Huth JF, Beitsch PD, Leitch M, Hoover S, Euhus D, et al: Circulating tumor cells in patients with breast cancer dormancy. Clin Cancer Res 10: 8152-8162, 2004

20. Watanabe S: The metastasizability of tumor cells. Cancer 7 : 215-223, 1954.

21. Garvie WH and Matheson AB: The effect of intravenous fluids on the development on experimental tumour metastases: Their effect on tumour cell aggregation. Br J Cancer 20: 838-846, 1966.

22. Topal B, Roskams T, Fevery J and Penninckx F: Aggregated colon cancer cells have a higher metastatic efficiency in the liver compared with nonaggregated cells: An experimental study. J Surg Res 112: 31-37, 2003.

23. Knisely WH and Mahaley MS Jr: Relationship between size and distribution of spontaneous metastases and three sizes of intravenously injected particles of VX2 carcinoma. Cancer Res 18: 900-905, 1958

24. Peeters DJ, Brouwer A, Van den Eynden GG, Rutten A, Onstenk W, Sieuwerts AM, Van Laere SJ, Huget P, Pauwels P, Peeters M, et al: Circulating tumour cells and lung microvascular tumour cell retention in patients with metastatic breast and cervical cancer. Cancer Lett 356B: 872-879, 2015.

25. Glaves D: Correlation between circulating cancer cells and incidence of metastases. Br J Cancer 48: 665-673, 1983.

26. Stott SL, Hsu CH, Tsukrov DI, Yu M, Miyamoto DT, Waltman BA, Rothenberg SM, Shah AM, Smas ME, Korir GK, et al: Isolation of circulating tumor cells using a microvortexgenerating herringbone-chip. Proc Natl Acad Sci USA 107: 18392-18397, 2010.

27. Duda DG, Duyverman AM, Kohno M, Snuderl M, Steller EJ, Fukumura D and Jain RK: Malignant cells facilitate lung metastasis by bringing their own soil. Proc Natl Acad Sci USA 107: 21677-21682, 2010.

28. Upreti M, Jamshidi-Parsian A, Koonce NA, Webber JS Sharma SK, Asea AA, Mader MJ and Griffin RJ: Tumorendothelial cell three-dimensional spheroids: New aspects to enhance radiation and drug therapeutics. Transl Oncol 4: 365-376, 2011

29. Hou JM, Krebs M, Ward T, Sloane R, Priest L, Hughes A, Clack G, Ranson M, Blackhall F and Dive C: Circulating tumor cells as a window on metastasis biology in lung cancer. Am J Pathol 178: 989-996, 2011.

30. Friedlander TW, Ngo VT, Dong H, Premasekharan G, Weinberg V, Doty S, Zhao Q, Gilbert EG, Ryan CJ, Chen WT, et al: Detection and characterization of invasive circulating tumor cells derived from men with metastatic castration-resistant prostate cancer. Int J Cancer 134: 2284-2293, 2014.

31. Balasubramanian P, Lang JC, Jatana KR, Miller B, Ozer E, Old M, Schuller DE, Agrawal A, Teknos TN, Summers TA Jr, et al: Multiparameter analysis, including EMT markers, on negatively enriched blood samples from patients with squamous cell carcinoma of the head and neck. PLoS One 7: e42048, 2012.
32. Brandt B, Junker R, Griwatz C, Heidl S, Brinkmann O, Semjonow A, Assmann G and Zänker KS: Isolation of prostatederived single cells and cell clusters from human peripheral blood. Cancer Res 56: 4556-4561, 1996.

33. Molnar B, Ladanyi A, Tanko L, Sréter L and Tulassay Z: Circulating tumor cell clusters in the peripheral blood of colorectal cancer patients. Clin Cancer Res 7: 4080-4085, 2001.

34. Wang ZP, Eisenberger MA, Carducci MA, Partin AW, Scher HI and Ts'o PO: Identification and characterization of circulating prostate carcinoma cells. Cancer 88: 2787-2795, 2000.

35. Sarioglu AF, Aceto N, Kojic N, Donaldson MC, Zeinali M, Hamza B, Engstrom A, Zhu H, Sundaresan TK, Miyamoto DT, et al: A microfluidic device for label-free, physical capture of circulating tumor cell clusters. Nat Methods 12: 685-691, 2015.

36. Vona G, Sabile A, Louha M, Sitruk V, Romana S, Schütze K, Capron F, Franco D, Pazzagli M, Vekemans M, et al: Isolation by size of epithelial tumor cells: A new method for the immunomorphological and molecular characterization of circulating tumor cells. Am J Pathol 156: 57-63, 2000.

37. Vona G, Estepa L, Béroud C, Damotte D, Capron F, Nalpas B, Mineur A, Franco D, Lacour B, Pol S, et al: Impact of cytomorphological detection of circulating tumor cells in patients with liver cancer. Hepatology 39: 792-797, 2004.

38. Krebs MG, Hou JM, Sloane R, Lancashire L, Priest L, Nonaka D, Ward TH, Backen A, Clack G, Hughes A, et al: Analysis of circulating tumor cells in patients with non-small cell lung cancer using epithelial marker-dependent and -independent approaches. J Thorac Oncol 7: 306-315, 2012.

39. Adams AA, Okagbare PI, Feng J, Hupert ML, Patterson D, Göttert J, McCarley RL, Nikitopoulos D, Murphy MC and Soper SA: Highly efficient circulating tumor cell isolation from whole blood and label-free enumeration using polymer-based microfluidics with an integrated conductivity sensor. J Am Chem Soc 130: 8633-8641, 2008.

40. Gleghorn JP, Pratt ED, Denning D, Liu H, Bander NH, Tagawa ST, Nanus DM, Giannakakou PA and Kirby BJ: Capture of circulating tumor cells from whole blood of prostate cancer patients using geometrically enhanced differential immunocapture (GEDI) and a prostate-specific antibody. Lab Chip 10: 27-29, 2010.

41. Mohamed H, Murray M, Turner JN and Caggana M: Isolation of tumor cells using size and deformation. J Chromatogr A 1216: 8289-8295, 2009.

42. Tan SJ, Yobas L, Lee GY, Ong CN and Lim CT: Microdevice for the isolation and enumeration of cancer cells from blood. Biomed Microdevices 11: 883-892, 2009.

43. Xu Y, Phillips JA, Yan J, Li Q, Fan ZH and Tan W: Aptamerbased microfluidic device for enrichment, sorting, and detection of multiple cancer cells. Anal Chem 81: 7436-7442, 2009.

44. Zheng S, Lin H, Liu JQ, Balic M, Datar R, Cote RJ and Tai YC: Membrane microfilter device for selective capture, electrolysis and genomic analysis of human circulating tumor cells. J Chromatogr A 1162: 154-161, 2007.

45. Hou HW, Warkiani ME, Khoo BL, Li ZR, Soo RA, Tan DS Lim WT, Han J, Bhagat AA and Lim CT: Isolation and retrieval of circulating tumor cells using centrifugal forces. Sci Rep 3: $1259,2013$.

46. Harouaka RA, Zhou MD, Yeh YT, Khan WJ, Das A, Liu X, Christ CC, Dicker DT, Baney TS, Kaifi JT, et al: Flexible micro spring array device for high-throughput enrichment of viable circulating tumor cells. Clin Chem 60: 323-333, 2014.

47. Cho EH, Wendel M, Luttgen M, Yoshioka C, Marrinucci D, Lazar D, Schram E, Nieva J, Bazhenova L, Morgan A, et al: Characterization of circulating tumor cell aggregates identified in patients with epithelial tumors. Phys Biol 9: 016001, 2012.

48. Ge F, Zhang H, Wang DD, Li L and Lin PP: Enhanced detection and comprehensive in situ phenotypic characterization of circulating and disseminated heteroploid epithelial and glioma tumor cells. Oncotarget 6: 27049-27064, 2015.

49. Zhang Y, Wang F, Ning N, Chen Q, Yang Z, Guo Y, Xu D, Zhang D, Zhan T and Cui W: Patterns of circulating tumor cells identified by CEP8, CK and CD45 in pancreatic cancer. Int J Cancer 136: 1228-1233, 2015

50. Zamay GS, Kolovskaya OS, Zamay TN, Glazyrin YE, Krat AV, Zubkova O, Spivak E, Wehbe M, Gargaun A, Muharemagic D, et al: Aptamers selected to postoperative lung adenocarcinoma detect circulating tumor cells in human blood. Mol Ther 23: $1486-1496,2015$ 
51. King MR, Phillips KG, Mitrugno A, Lee TR, de Guillebon AM, Chandrasekaran S, McGuire MJ, Carr RT, Baker-Groberg SM, Rigg RA, et al: A physical sciences network characterization of circulating tumor cell aggregate transport. Am J Physiol Cell Physiol 308: C792-C802, 2015.

52. Cheung KJ, Padmanaban V, Silvestri V, Schipper K, Cohen JD, Fairchild AN, Gorin MA, Verdone JE, Pienta KJ, Bader JS et al: Polyclonal breast cancer metastases arise from collective dissemination of keratin 14-expressing tumor cell clusters. Proc Natl Acad Sci USA 113: E854-E863, 2016.

53. Glinsky VV, Glinsky GV, Glinskii OV, Huxley VH, Turk JR, Mossine VV, Deutscher SL, Pienta KJ and Quinn TP: Intravascular metastatic cancer cell homotypic aggregation at the sites of primary attachment to the endothelium. Cancer Res 63 : 3805-3811, 2003

54. Geng Y, Chandrasekaran S, Hsu JW, Gidwani M, Hughes AD and King MR: Phenotypic switch in blood: Effects of proinflammatory cytokines on breast cancer cell aggregation and adhesion. PLoS One 8: e54959, 2013.

55. Fidler IJ: Immune stimulation-inhibition of experimental cancer metastasis. Cancer Res 34: 491-498, 1974.

56. Gasic GJ, Gasic TB, Galanti N, Johnson T and Murphy S: Platelet-tumor-cell interactions in mice. The role of platelets in the spread of malignant disease. Int J Cancer 11: 704-718, 1973.

57. Läubli H, Stevenson JL, Varki A, Varki NM and Borsig L: L-selectin facilitation of metastasis involves temporal induction of Fut7-dependent ligands at sites of tumor cell arrest. Cancer Res 66: 1536-1542, 2006

58. Küsters B, Kats G, Roodink I, Verrijp K, Wesseling P, Ruiter DJ, de Waal RM and Leenders WP: Micronodular transformation as a novel mechanism of VEGF-A-induced metastasis. Oncogene 26 5808-5815, 2007.

59. Borsig L, Wong R, Hynes RO, Varki NM and Varki A: Synergistic effects of L- and P-selectin in facilitating tumor metastasis can involve non-mucin ligands and implicate leukocytes as enhancers of metastasis. Proc Natl Acad Sci USA 99: 2193-2198, 2002.

60. Molnar B, Floro L, Sipos F, Toth B, Sreter L and Tulassay Z: Elevation in peripheral blood circulating tumor cell number correlates with macroscopic progression in UICC stage IV colorectal cancer patients. Dis Markers 24: 141-150, 2008.

61. Sugino T, Kusakabe T, Hoshi N, Yamaguchi T, Kawaguchi T, Goodison S, Sekimata M, Homma Y and Suzuki T: An invasionindependent pathway of blood-borne metastasis: A new murine mammary tumor model. Am J Pathol 160: 1973-1980, 2002.

62. Ao Z, Shah SH, Machlin LM, Parajuli R, Miller PC, Rawal S, Williams AJ, Cote RJ, Lippman ME, Datar RH, et al: Identification of cancer-associated fibroblasts in circulating blood from patients with metastatic breast cancer. Cancer Res 75: 4681-4687, 2015

63. Yu M, Bardia A, Wittner BS, Stott SL, Smas ME, Ting DT, Isakoff SJ, Ciciliano JC, Wells MN, Shah AM, et al: Circulating breast tumor cells exhibit dynamic changes in epithelial and mesenchymal composition. Science 339: 580-584, 2013.

64. Sharma D, Brummel-Ziedins KE, Bouchard BA and Holmes CE Platelets in tumor progression: A host factor that offers multiple potential targets in the treatment of cancer. J Cell Physiol 229: $1005-1015,2014$

65. Lecharpentier A, Vielh P, Perez-Moreno P, Planchard D, Soria JC and Farace F: Detection of circulating tumour cells with a hybrid (epithelial/mesenchymal) phenotype in patients with metastatic non-small cell lung cancer. Br J Cancer 105: 1338-1341, 2011.

66. Maddipati R and Stanger BZ: Pancreatic cancer metastases harbor evidence of polyclonality. Cancer Discov 5: 1086-1097, 2015.

67. Au SH, Storey BD, Moore JC, Tang Q, Chen YL, Javaid S, Sarioglu AF, Sullivan R, Madden MW, O'Keefe R, et al: Clusters of circulating tumor cells traverse capillary-sized vessels. Proc Natl Acad Sci USA 113: 4947-4952, 2016

68. Fidler IJ and Talmadge JE: Evidence that intravenously derived murine pulmonary melanoma metastases can originate from the expansion of a single tumor cell. Cancer Res 46: 5167-5171, 1986

69. Zhao Q, Barclay M, Hilkens J, Guo X, Barrow H, Rhodes JM and Yu LG: Interaction between circulating galectin-3 and cancerassociated MUC1 enhances tumour cell homotypic aggregation and prevents anoikis. Mol Cancer 9: 154, 2010.

70. Zhao Q, Guo X, Nash GB, Stone PC, Hilkens J, Rhodes JM and Yu LG: Circulating galectin-3 promotes metastasis by modifying MUC1 localization on cancer cell surface. Cancer Res 69: 6799-6806, 2009.
71. Yu LG, Andrews N, Zhao Q, McKean D, Williams JF, Connor LJ, Gerasimenko OV, Hilkens J, Hirabayashi J, Kasai K, et al: Galectin-3 interaction with Thomsen-Friedenreich disaccharide on cancer-associated MUC1 causes increased cancer cell endothelial adhesion. J Biol Chem 282: 773-781, 2007.

72. Labelle M, Begum S and Hynes RO: Direct signaling between platelets and cancer cells induces an epithelial-mesenchymal-like transition and promotes metastasis. Cancer Cell 20: 576-590, 2011.

73. Wels J, Kaplan RN, Rafii S and Lyden D: Migratory neighbors and distant invaders: Tumor-associated niche cells. Genes Dev 22: 559-574, 2008.

74. Psaila B and Lyden D: The metastatic niche: Adapting the foreign soil. Nat Rev Cancer 9: 285-293, 2009.

75. Phillips KG, Lee AM, Tormoen GW, Rigg RA, Kolatkar A, Luttgen M, Bethel K, Bazhenova L, Kuhn P, Newton P, et al: The thrombotic potential of circulating tumor microemboli: Computational modeling of circulating tumor cell-induced coagulation. Am J Physiol Cell Physiol 308: C229-C236, 2015.

76. Denes V, Lakk M, Makarovskiy A, Jakso P, Szappanos S, Graf L, Mandel L, Karadi I and Geck P: Metastasis blood test by flow cytometry: In vivo cancer spheroids and the role of hypoxia. Int J Cancer 136: 1528-1536, 2015

77. Al-Mehdi AB, Tozawa K, Fisher AB, Shientag L, Lee A and Muschel RJ: Intravascular origin of metastasis from the proliferation of endothelium-attached tumor cells: A new model for metastasis. Nat Med 6: 100-102, 2000.

78. Mu Z, Wang C, Ye Z, Austin L, Civan J, Hyslop T, Palazzo JP, Jaslow R, Li B, Myers RE, et al: Prospective assessment of the prognostic value of circulating tumor cells and their clusters in patients with advanced-stage breast cancer. Breast Cancer Res Treat 154: 563-571, 2015.

79. Jansson S, Bendahl PO, Larsson AM, Aaltonen KE and Rydén L: Prognostic impact of circulating tumor cell apoptosis and clusters in serial blood samples from patients with metastatic breast cancer in a prospective observational cohort. BMC Cancer 16: 433, 2016.

80. Mascalchi M, Falchini M, Maddau C, Salvianti F, Nistri M, Bertelli E, Sali L, Zuccherelli S, Vella A, Matucci M, et al: Prevalence and number of circulating tumour cells and microemboli at diagnosis of advanced NSCLC. J Cancer Res Clin Oncol 142: 195-200, 2016

81. Giesing M, Suchy B, Driesel G and Molitor D: Clinical utility of antioxidant gene expression levels in circulating cancer cell clusters for the detection of prostate cancer in patients with prostate-specific antigen levels of $4-10 \mathrm{ng} / \mathrm{ml}$ and disease prognostication after radical prostatectomy. BJU Int 105: 1000-1010, 2010.

82. Choi JW, Kim JK, Yang YJ, Kim P, Yoon KH and Yun SH: Urokinase exerts antimetastatic effects by dissociating clusters of circulating tumor cells. Cancer Res 75: 4474-4482, 2015.

83. Wendel M, Bazhenova L, Boshuizen R, Kolatkar A, Honnatti M, Cho EH, Marrinucci D, Sandhu A, Perricone A, Thistlethwaite P, et al: Fluid biopsy for circulating tumor cell identification in patients with early-and late-stage non-small cell lung cancer: A glimpse into lung cancer biology. Phys Biol 9: 016005, 2012.

84. Reddy RM, Murlidhar V, Zhao L, Grabauskiene S, Zhang Z, Ramnath N, Lin J, Chang AC, Carrott P, Lynch W, et al: Pulmonary venous blood sampling significantly increases the yield of circulating tumor cells in early-stage lung cancer. J Thorac Cardiovasc Surg 151: 852-857, 2016.

85. Kats-Ugurlu G, Roodink I, de Weijert M, Tiemessen D, Maass C, Verrijp K, van der Laak J, de Waal R, Mulders P, Oosterwijk E, et al: Circulating tumour tissue fragments in patients with pulmonary metastasis of clear cell renal cell carcinoma. J Pathol 219: 287-293, 2009.

86. Loh J, Jovanovic L, Lehman M, Capp A, Pryor D, Harris M, Nelson C and Martin J: Circulating tumor cell detection in high-risk non-metastatic prostate cancer. J Cancer Res Clin Oncol 140: 2157-2162, 2014.

87. Chang MC, Chang YT, Chen JY, Jeng YM, Yang CY, Tien YW, Yang SH, Chen HL, Liang TY, Wang CF, et al: Clinical significance of circulating tumor microemboli as a prognostic marker in patients with pancreatic ductal adenocarcinoma. Clin Chem 62: 505-513, 2016.

88. Long E, Ilie M, Bence C, Butori C, Selva E, Lalvée S, Bonnetaud C, Poissonnet G, Lacour JP, Bahadoran P, et al: High expression of TRF2, SOX10, and CD10 in circulating tumor microemboli detected in metastatic melanoma patients. A potential impact for the assessment of disease aggressiveness. Cancer Med 5: 1022-1030, 2016. 